\title{
Elastographic Region of Interest Determination for Muscle with Fat Infiltration
}

\author{
Takuma Yuri (iD) ${ }^{1,2}$ \\ Nariyuki Mura 1,3,4 \\ Kyosuke Hoshikawa ${ }^{4}$ \\ Hugo Giambini ${ }^{2}$ \\ Hiromi Fujii \\ Yoshiro Kiyoshige (iD) 1,4 \\ 'Graduate School of Health Sciences, \\ Yamagata Prefectural University of Health \\ Sciences, Yamagata, Japan; ${ }^{2}$ Department \\ of Biomedical Engineering, The University \\ of Texas at San Antonio, San Antonio, \\ TX, USA; ${ }^{3}$ Department of Orthopaedic \\ Surgery, Yoshioka Hospital, Tendo, Japan; \\ ${ }^{4}$ Department of Physical Therapy, \\ Yamagata Prefectural University of Health \\ Sciences, Yamagata, Japan
}

Correspondence: Takuma Yuri Graduate School of Health Sciences, Yamagata Prefectural University of Health Sciences, 260 Kamiyanagi, Yamagata, 990-2212, Japan Tel +8I-23-686-674l

Emaild2167013@yachts.ac.jp
Purpose: Ultrasound elastography has been used to evaluate the skeletal muscle stiffness as a biomarker for sarcopenia assessment. However, there is no consensus with respect to the size and location of the region of interest in assessing such fat infiltrated muscle. The objective of this study was to determine which cross-sectional area should be measured in torn disuse muscle with fat infiltration to accurately measure muscle activity using real-time tissue elastography (RTE).

Methods: Twenty-seven patients, whose rotator cuff muscle with torn tendon was successfully repaired, were followed by programmed rehabilitation. RTE measurements of the supraspinatus muscle were obtained during muscle contraction before and one-year after surgery so that the activity value was defined as the difference between elastography measurements at rest and elastography measurements during contraction. Given that the patients with successfully repaired and completed rehabilitation showed an increased activity value, the sensitivity for three regions of interest; posterior portion of the anterior-middle subregion (AM-p), anterior region (AR), and whole cross-sectional area of the supraspinatus (whole) were compared with the number of patients showing an increase in activity values as sensitivity analysis.

Results: The sensitivity showing an increase in activity values was $74.1 \%$ for the AM-p area, $70.4 \%$ for the $\mathrm{AR}$ area, and $81.5 \%$ for the whole area. Intraclass correlation coefficient $_{1,3}$ was $0.87-0.97$ for the AM-p area, $0.88-0.98$ for the AR area and 0.92-0.99 for the whole area.

Conclusion: The whole cross-sectional area is suitable to measure muscle activity in muscle with fat infiltration. The results in this study will provide some beneficial information when ultrasound elastography is used for the assessment of sarcopenia muscle with fat infiltration. Keywords: ultrasound elastography, region of interest, sarcopenia, fat infiltration, supraspinatus muscle

\section{Introduction}

Sarcopenia is defined as loss of muscle mass and strength, and sarcopenia muscle is characterized as not only muscle atrophy but also fat infiltration. ${ }^{1}$ Magnetic resonance imaging, which is a suitable tool for evaluating sarcopenia muscle pathology, has

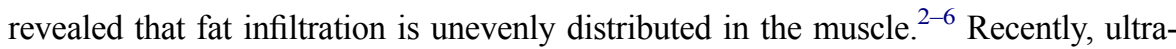
sound elastography has also been used to evaluate the skeletal muscle passive stiffness and muscle activity as a biomarker for sarcopenia assessment. ${ }^{7,8}$ However, there is no consensus with respect to the size and location of the region of interest (ROI) in assessing such fat infiltrated muscle. In healthy muscle, Ates et al found that the \% error in muscle stiffness during contraction between full ROI and that of $1 / 2,1 / 4$, and 
$1 / 16$ increased with decreasing ROI size. ${ }^{9}$ Other studies also found a significant effect of ROI size on muscle stiffness. ${ }^{10,11}$ They suggested that the size of ROI should be set as large as possible so that meaningful ultrasound elastography outcomes can be obtained. ${ }^{9-11}$ Sendur et al revealed that muscle stiffness decreased in the elder's muscle compared with younger's one. ${ }^{8}$ In sarcopenia muscle, fat infiltration is unevenly distributed. ${ }^{3,6,12}$ Kiyoshige et al and Takano et al found that the localization of fatty tissue in the anterior part of the gluteus minimus muscle. ${ }^{3,6}$ Rosatelli et al reported that fat infiltration localized at the bony side in the multifidus muscle. ${ }^{13}$ The localization of fat infiltration in skeletal muscle should be considered when we set the ROI because the fatty tissue generally presenting as a soft elasticity outcome, and the tendinous tissue, if it is included in the ROI, generally showing as a hard elasticity outcome compared to muscle fibers.

In order to prevent increasing in sarcopenia, resistance exercise is recommended. ${ }^{14}$ The cause of sarcopenia is multifactorial. However, its muscular histopathology, namely disuse degeneration such as fat infiltration, is common with that of torn and retracted muscle. Muscle degeneration in sarcopenia is systemic, while that in torn and retracted muscle is local. Rotator cuff muscle injury is common in middle and aged manual workers. The treatment of the torn rotator cuff muscle has been established. The resistance exercise was gradually adapted along with a welldeveloped protocol after torn rotator cuff tendon was repaired, and obtained excellent outcomes. When the torn rotator cuff muscle is repaired, the programmed rehabilitation is followed. Constant interventional outcomes can be obtained in the treatment of the torn rotator cuff muscle, while the outcomes in resistance exercise for the elderly may be varied because the individual pre-interventional physical condition and the way of intervention are various in the sarcopenia elders. To take the torn rotator cuff muscle to the subject as a fat infiltrated muscle will be beneficial for assessing interventional outcomes rather than the muscle of sarcopenia elders. Ultrasound elastography evaluation before and after the surgery and rehabilitation of the torn rotator cuff muscle provides useful information in the recovery of fat infiltrated muscle contractility. The aim of current study is to determine which area is adequate for setting the region of interest on the basis of recovery of contractility in the torn supraspinatus muscle.

\section{Materials and Methods}

\section{Subjects}

Thirty-five patients who were scheduled for arthroscopic rotator cuff repair were evaluated using real-time tissue elastography (RTE) and magnetic resonance imaging (MRI) before and one year after surgery. RTE assessments were performed immediately before or after MRI assessments. The study cohort consisted of 23 males and 12 females and their mean age was $67 \pm 8$ yrs (range 49-83). The inclusion criteria for this study are a successfully repaired tendon defined as the patients who did not show any presence of discontinuity in the coronal plane of the MRI one-year after surgery. Eight patients in whom the presence of discontinuity was found were excluded from further analyses and evaluations, because their outcomes did not fit our hypothesis that muscle activity, in patients with a successful repair of the supraspinatus tendon, would show an increase in muscle activity. Thus, the total number of patients in this study was comprised of 27 patients (Figure 1).

MRI assessment was conducted by a licensed orthopedic surgeon with 30 years of experience in orthopedic MRI, who was blinded to the RTE assessment. Fat infiltration of the supraspinatus muscle was evaluated on the sagittal Y-view of the MRI before and one-year after surgery using the Fuchs' modified Goutallier stage: ${ }^{15,16}$ stage 0 , normal muscle without any fatty streaks; stage 1 , the muscle contains some fatty streaks; stage 2, fat infiltration is significant but

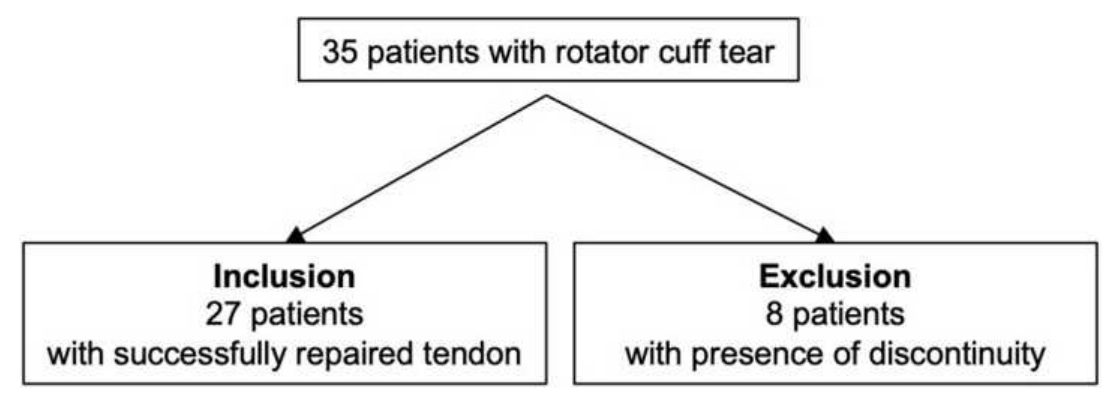

Figure I Diagram for patient inclusion. 
there is still more muscle than fat; stage 3 , there is as much fat as muscle; and stage 4, more fat than muscle is present.

\section{Muscle Activity Measurement by Real-Time Tissue Elastography}

RTE assessment was performed by a licensed occupational therapist with 5 years of experience in shoulder ultrasound elastography, who was blinded to the Fuchs' modified Goutallier stage, before and one-year after surgery. The patients were instructed to sit on a chair with the affected arm resting on a table with the shoulder abducted to $60^{\circ}$ and with neutral rotation in the scapular plane. ${ }^{17}$ RTE measurements were obtained with the arm resting on the table and during muscle contraction, accomplished by having the patient hold the affected arm $1 \mathrm{~cm}$ against gravity for approximately ten seconds. We used a diagnostic ultrasound system (Noblus; Hitachi-Aloka Medical Japan, Tokyo, Japan) with a linear array probe (L-64; Hitachi-Aloka Medical Japan) combined with an acoustic coupler (EZU-TECPL1; HitachiAloka Medical Japan). ${ }^{18}$ We oriented the cross-sectional ultrasound B-mode images by placing the probe $2 \mathrm{~cm}$ medial to the acromion process and obtained the RTE images by performing cyclic manual compression with the probe. The RTE measurements took less than 10 minutes.

Three elastography images were randomly selected for each condition, rest and contraction, for analyses. Muscle hardness was calculated as the strain ratio (SR), which is the relative value in reference to the acoustic coupler of 22.6 $\pm 2.2 \mathrm{kPa}$. A higher $\mathrm{SR}$ value represents softer tissue and a lower SR value represents harder tissue. We defined 2 SR values: SR value at rest and SR value at contraction. Because the former represents the muscle hardness itself and the latter represents the sum of the muscle hardness at rest and the hardness produced by contraction, we defined the difference between the SR value at rest and the SR value at contraction as the activity value. Three ROIs, namely posterior portion of the anterior-middle subregion (AM-p), anterior region (AR), and whole cross-sectional area (whole) of supraspinatus muscle were defined as follows: $A M-p$ includes muscle fibers posterior to intramuscular tenon in anterior-middle subregion defined by Kim et $\mathrm{al}^{19}$ without intramuscular tendon and obvious fatty streaks; $A R$, is responsible for contractile function $^{19-21}$ including intramuscular tendon and fat tissue to some extent; whole, includes whole cross-sectional area of the supraspinatus muscle to account for the previous research and recommendations $^{9-11}$ (Figure 2).

\section{Statistics}

SPSS statistical software (version 24.0; SPSS, Chicago, IL, USA) was used for all data analyses. After a non-normal distribution visualization of the data, as indicated by the Shapiro-Wilk test, non-parametric tests were performed on the data outcomes. The Kruskal-Wallis test, followed by the Bonferroni post hoc test, was used to investigate the

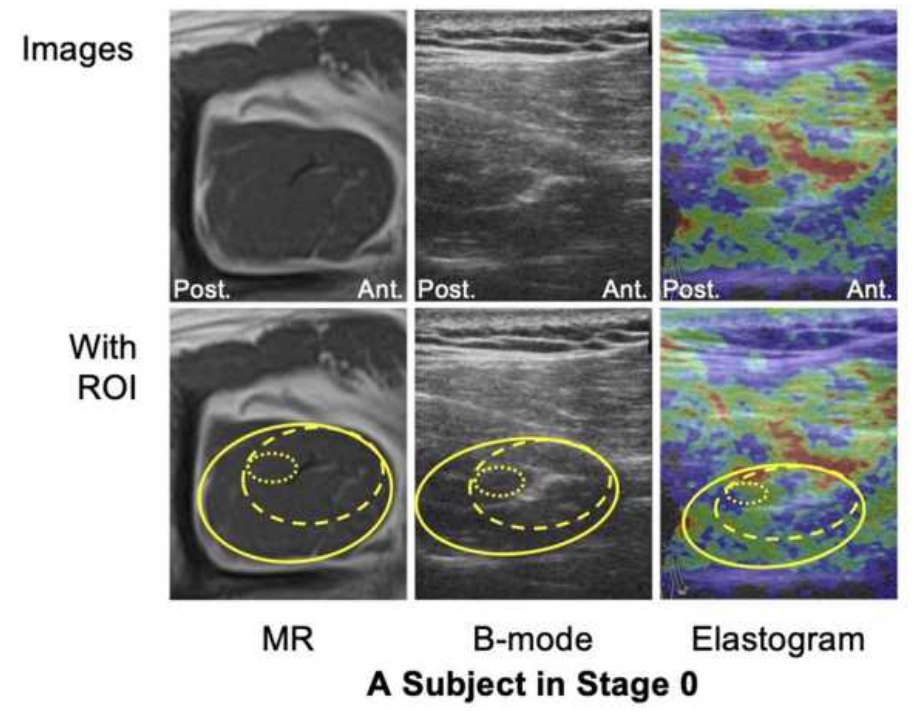

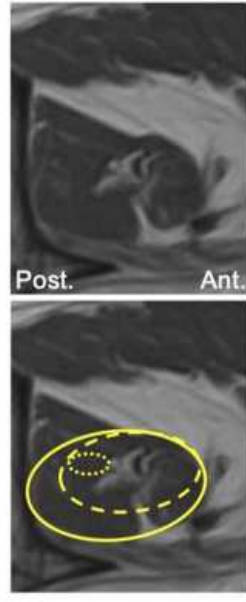

MR

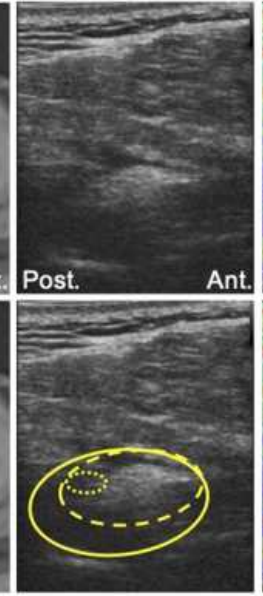

B-mode

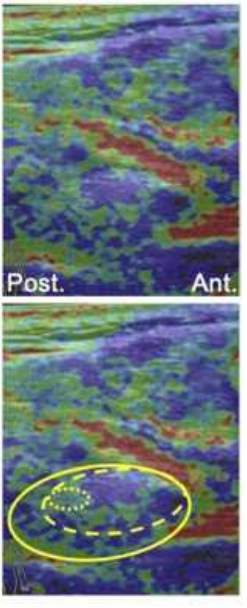

Elastogram

A Subject in Stage 2

Figure 2 The cross-sectional measurement areas of the AM-p (the dotted circler area), AR (the dashed circler area) and whole (the solid circler area) of the supraspinatus. Notes: The cross-sectional area in the AM-p can be measured residual muscle fibers excluding the internal tendon and fatty tissue. The AR area is responsible for contractility while included the internal tendon and fat infiltration to some extent. The whole area is occupied by a higher proportion of the residual muscle fibers even though the area contains a harder internal tendon and softer fatty tissue.

Abbreviations: AM-p, posterior part of the anterior-middle subregion; AR, anterior region; MR, magnetic resonance; ROI, region of interest; Ant., anterior side; Post., posterior side. 
difference among the Goutallier stages in all measurement areas. In addition, Friedman with Dunn's post hoc tests was used to investigate the difference among the three measured areas. Given that the patients with successfully repaired tendon showed an increased activity value, ${ }^{22}$ the sensitivity for the AM-p, AR, and whole measurements were described as the number of patients showing an increase in activity values. The sensitivity for the AM-p, AR, and whole measurements were also calculated in subgroup analysis for age ( $<65$ yrs. versus $>65$ yrs.), gender (male versus female), and side of supraspinatus tear (dominant versus non-dominant). The elastography images for a representative patient showing an increased activity value before and after surgery is shown in Figure 3. Intraclass correlation coefficient $\left(\mathrm{ICC}_{1,3}\right)$ analysis was also performed for all three measurements to examine intra-tester reliability. $P$ values lower than 0.05 were considered statistically significant.

\section{Ethics}

Written informed consent to participate in this study was obtained from all patients. Ethical approval was obtained from the Ethics Committee of Yamagata Prefectural University of Health Sciences (\#1705-01). This study was conducted in accordance with the Declaration of Helsinki.

\section{Results}

Fat infiltration evaluations of the supraspinatus muscle resulted in three patients with a stage 0,20 patients with a stage 1 , three patients with a stage 2 , and one patient with a stage 3. Measurements before and one year after surgery resulted in similar staging outcomes. Pre-operative SR values at rest and in all measurement ROIs decreased with increasing Goutallier stages (Figure 4). Post-operative SR values at rest also decreased with increasing Goutallier stages, with significant differences observed between patients with stage 2 and stage 1, with the former presenting lower values $(P=0.038$ for AM-p, $P=0.040$ for AR, and $P=0.050$ for whole, respectively). Pre- and post-operative SR values during contraction were not significantly different for all ROIs, except for the post-operative measurements using the whole ( $P=0.018$; Figure 4$)$.

A comparison between the SR outcomes from the three ROIs at rest showed that, pre- and post-operatively, the $A R$ measurement tended to present higher values, except for those in stage 2 post-operatively. Significant differences were found in patients with Goutallier stage $1(P=0.040$ for preoperative, and $P=0.007$ for post-operative measurements; Figure 5A). The SR value at contraction did not show any significant differences between all three ROIs (Figure 5B).

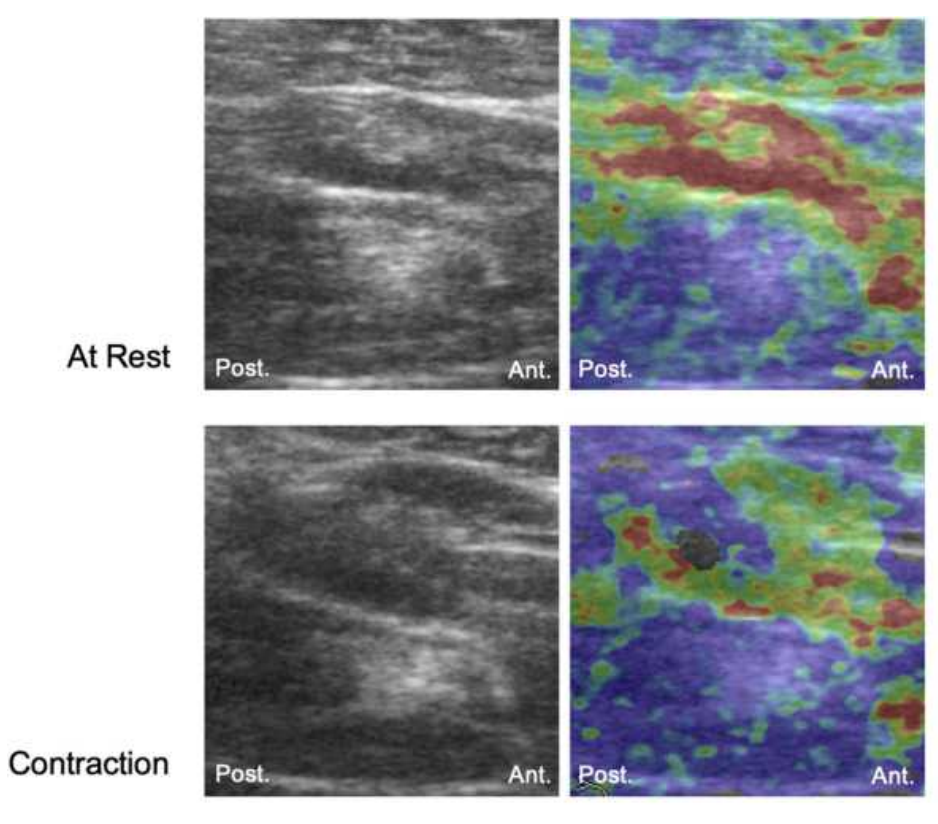

Before Surgery
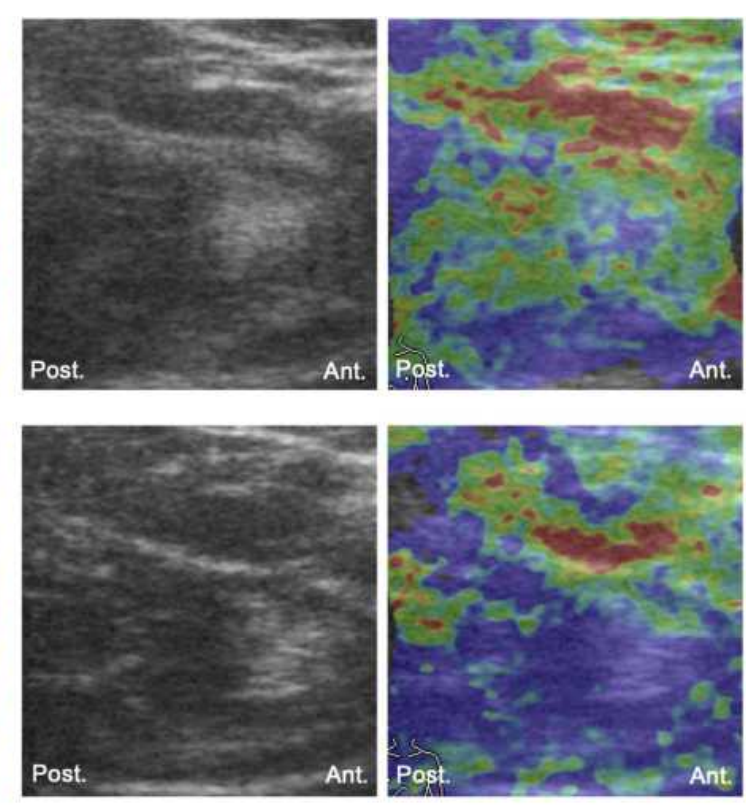

One Year After Surgery

Figure 3 Elastography images at rest and during contraction before and one year after surgery.

Notes: The elastogram at rest after surgery showed green in the supraspinatus muscle, while that before surgery indicated blue, which indicated that the elasticity in the supraspinatus muscle got softer after surgery than that before surgery.

Abbreviations: Ant., anterior side; Post., posterior side. 


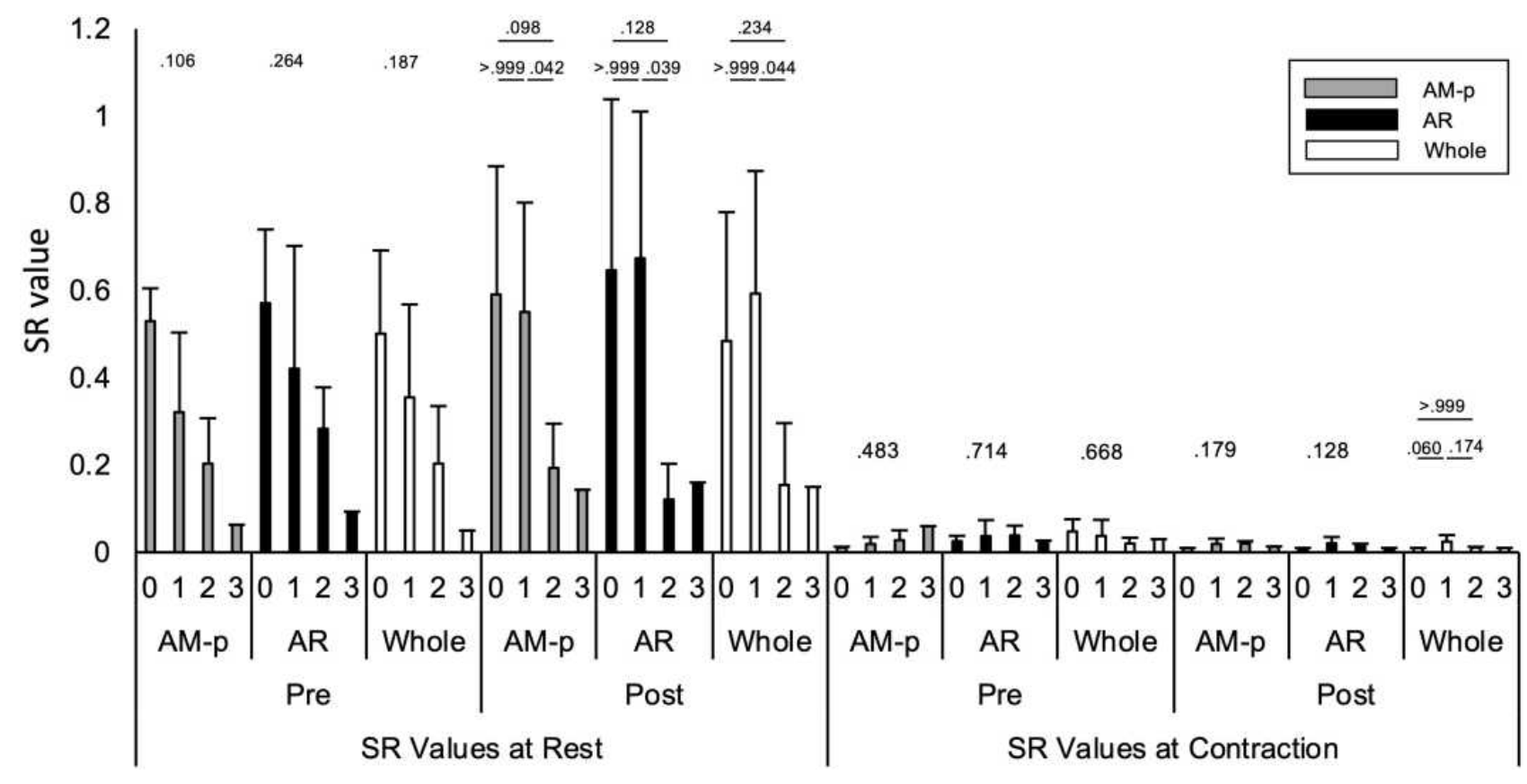

Figure 4 The pre- and post-operative SR value at rest and at contraction in relation to Goutallier stage.

Notes: Pre- and post-operative SR values at rest and in all measurement ROls decreased with increasing Goutallier stages. Those during contraction were not significantly different for all ROls, except for the post-operative measurements using the whole.

Abbreviations: AM-p, posterior part of the anterior-middle subregion; AR, anterior region; Pre, pre-operative measurement; Post, post-operative measurement; ROI, region of interest; SR, strain ratio.

An increase in activity values was found in 20 out of 27 patients with successfully repaired tendon when using the AM-p, in 19 patients using the AR, and in 22 when the whole was selected. A decrease in activity value was observed in 7,8, and 4 patients when implementing the AM-p, AR and whole, respectively (Figure 6A-C). One patient showed no change in pre- and post-operative activity values when using the whole and was considered as neither an increase nor decrease data value in our sensitivity analysis. The sensitivity for patients with successfully repaired tendon integrity showing an increase in activity values was $74.1 \%$ for the AM-p, $70.4 \%$ for the $\mathrm{AR}$, and $81.5 \%$ for the whole. For 7 patients with a Goutallier stage $\geq 2$, the sensitivity was $75.0 \%$ for the AM-p, $25.0 \%$ for the AR, and $50.0 \%$ for the whole. $\mathrm{ICC}_{1,3}$ was $0.87-0.97$ for the AM-p, $0.88-0.98$ for the AR and 0.92-0.99 for the whole, respectively (Table 1). An increase in SR values at rest was associated with an increase in activity values.

Subgroup analysis for age ( $<65$ yrs. versus $>65$ yrs.), gender (male versus female), and side of supraspinatus tear (dominant versus non-dominant) also showed the highest sensitivity for the whole measurement. For 14 patients aged less than 65 , the sensitivity was $71.4 \%$ for the AM-p, $78.6 \%$ for the AR, and $85.7 \%$ for the whole, respectively, while that was $76.9 \%$ for the AM-p, $61.5 \%$ for the AR, and $84.6 \%$ for the whole in the 13 patients with aged more than 65 . There were 16 males and 11 females in our participants. The sensitivity was $75.0 \%$ for the AM-p, $62.5 \%$ for the AR, and $75.0 \%$ for the whole, respectively, in the male group, whereas that was $72.7 \%$ for the AM-p, $81.8 \%$ for the AR, and $100 \%$ for the whole in the female group, respectively. The patients with right supraspinatus tear were 17, and that with the left was 10. The sensitivity was $76.5 \%$ for the AM-p, $82.4 \%$ for the $\mathrm{AR}$, and $94.1 \%$ for the whole in the patients with right side supraspinatus tear, while that was $70.0 \%$ for the AM-p, $50.0 \%$ for the AR, and $70.0 \%$ for the whole in the patients with the left side.

\section{Discussion}

Ultrasound elastography has been used to evaluate the skeletal muscle stiffness as a biomarker for sarcopenia assessment. ${ }^{7,8}$ However, there is no consensus with respect to the size and location of ROI in assessing such fat infiltrated muscle. In healthy muscle, it has been recommended that the ROI should be set as large as possible because the number of muscle fibers in the measurements increases with an increase in the size of ROI, ${ }^{9-11}$ and the error increases with a decrease in ROI size. ${ }^{9}$ In this study, we investigated 


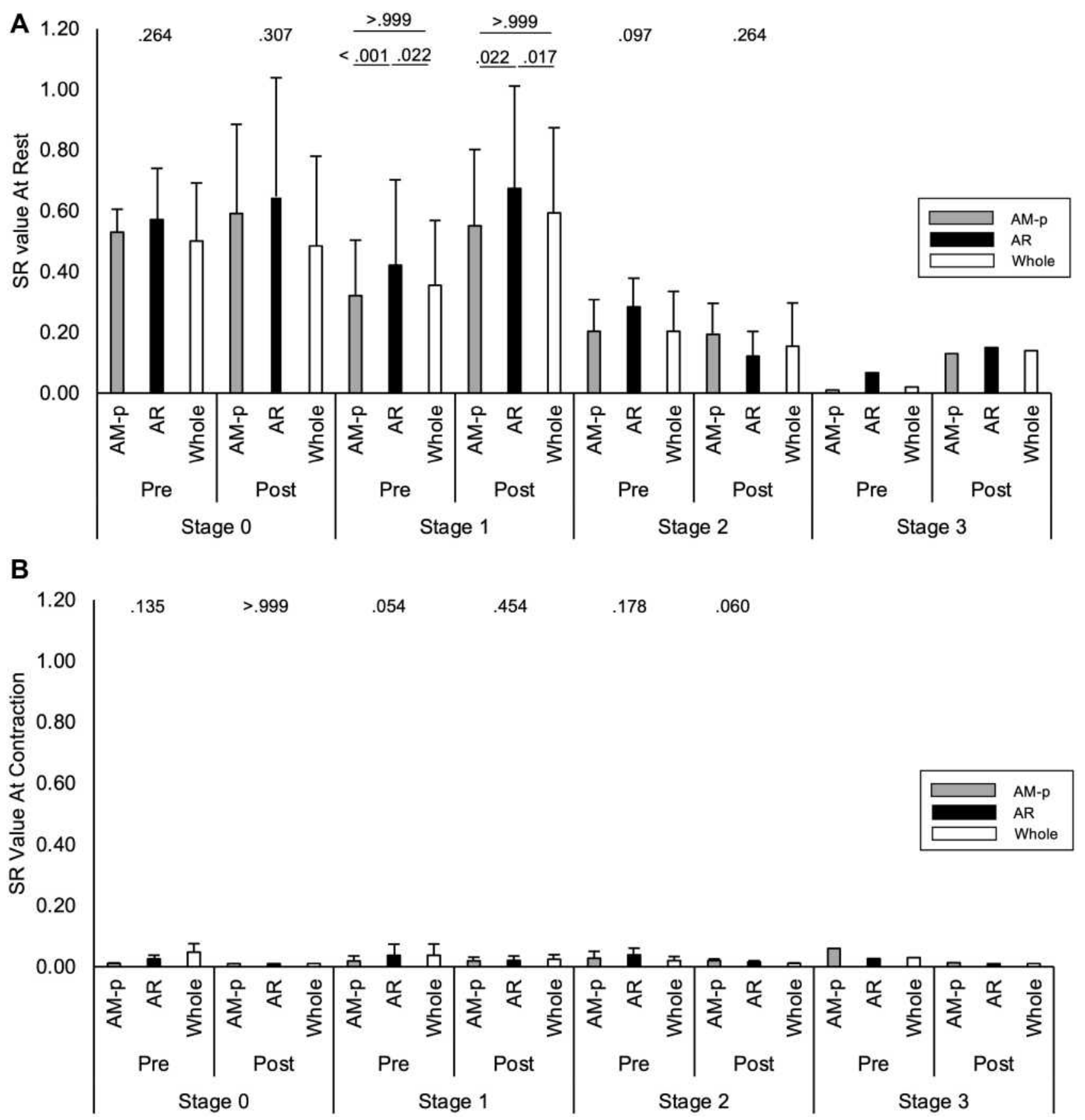

Figure 5 Comparison among three ROI measurement in pre- and post-operative SR value in relation to Goutallier stage.

Notes: (A) At rest. (B) At contraction.

Abbreviations: AM-p, posterior part of the anterior-middle subregion; AR, anterior region; Pre, pre-operative measurement; Post, post-operative measurement; ROI, region of interest; $S R$, strain ratio.

the effect of the ROI selection on activity outcomes in muscle with fat infiltration using RTE and found that selecting the whole supraspinatus cross-sectional area led to the highest sensitivity and reliability in outcomes as supporting the recommendation previously developed.

The AM-p area excluded the internal tendon and marked fat infiltration. The AR area was suggested to be responsible for contractility while included the internal tendon. The whole area included both the internal tendon and a part of marked fat infiltration. Many studies have revealed that elasticity decreased with increasing fat infiltration. ${ }^{17,23-25}$ Valencia et al demonstrated that interstitial fibrosis advanced with increasing fat infiltration, especially dense collagenous tissue surrounded the marked 
A

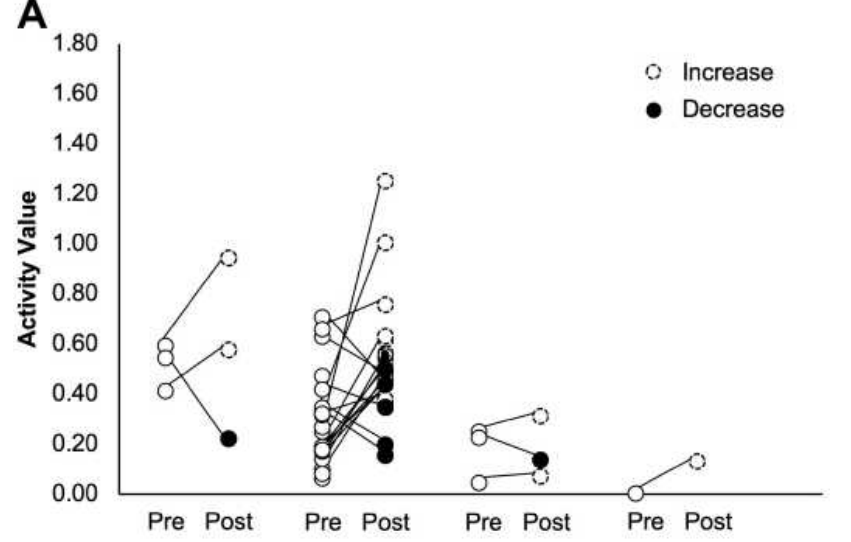

B

0

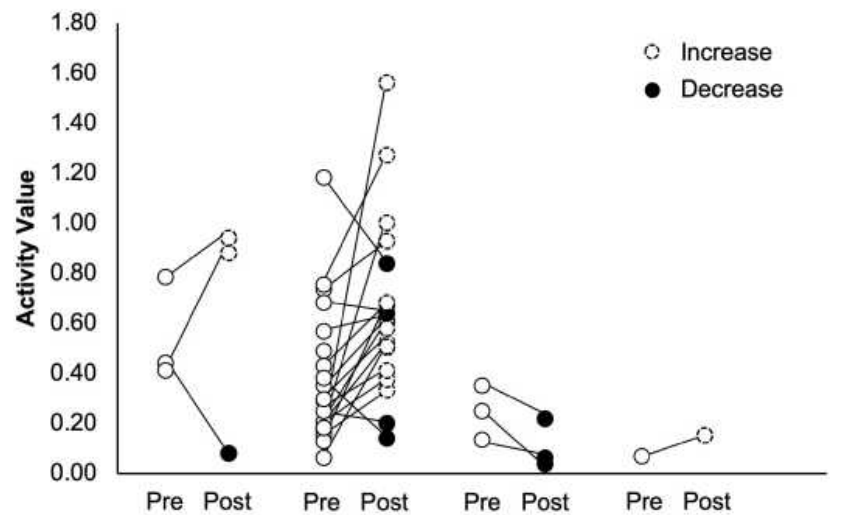

C

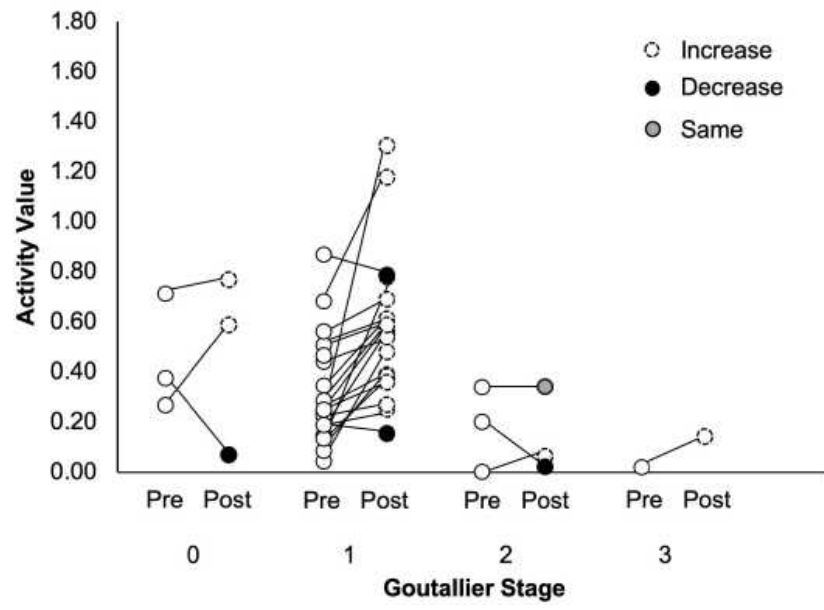

Figure 6 Pre- and post-operative activity value in relation to Goutallier stage. Notes: (A) Pre- and post-operative activity values measured by AM-p in relation to Goutallier stage. The activity value increases from pre- to post-operatively, except for one patient in stage 0 , five in stage I, and one in stage 2, respectively. (B) Preand post-operative activity values measured by AR in relation to Goutallier stage. The activity value increases from pre- to post-operatively, except for one patient in stage 0 , four in stage 1 , and 3 in stage 2 , respectively. (C) Pre- and post-operative activity values measured by Whole in relation to Goutallier stage. The activity value increases from pre- to post-operatively, except for one patient in stage 0 , two in stage $\mathrm{I}$, and one in stage 2, respectively. One patient in stage 2 showed the same activity value both pre- and post-operatively.

Abbreviations: AM-p, posterior part of the anterior-middle subregion; AR, anterior region; Pre, pre-operative measurement; Post, post-operative measurement.
Table I Sensitivity and Reliability in the Activity Value

\begin{tabular}{|c|c|c|c|}
\hline & AM-p & AR & Whole \\
\hline $\begin{array}{l}\text { Number of True- } \\
\text { Positive }\end{array}$ & $20(3)$ & $19(1)$ & $22(2)$ \\
\hline $\begin{array}{l}\text { Number of False- } \\
\text { Positive }\end{array}$ & $7(\mathrm{I})$ & $8(3)$ & $4(1)$ \\
\hline Sensitivity (\%) & $74.1(75.0)$ & $70.4(25.0)$ & $81.5(50.0)$ \\
\hline $\mathrm{ICC}_{1,3}$ & 0.87 to 0.97 & 0.88 to 0.98 & 0.92 to 0.99 \\
\hline
\end{tabular}

Notes: Values indicate the number of patients (patients with stage $\geq 2$ ). The patients whose strain ratio value at rest increased showed an increase in the activity value and the patient whose strain ratio value at rest decreased accompanied a decrease in the activity value. The activity value measured as whole in one patient showed same value pre- and post-operatively. Thus, the patient did not count in neither number of true-positive or false-positive.

Abbreviations: AM-p, posterior part of the anterior-middle subregion; AR, anterior region; ICC, intraclass correlation coefficient.

fat infiltration. ${ }^{26}$ Theoretically, the collagenous tissue content ratio will be highest in the AR area, modest in the whole area and lowest in the AM-p area.

Numerous studies revealed that poor surgical outcome obtained in advanced fat infiltrated rotator cuff muscle. ${ }^{16,27}$ Giambini et al demonstrated that the extensibility in muscle with fat infiltration decreased with increasing fat infiltration. ${ }^{23,24}$ Gerber et al and Yuri et al independently demonstrated that the contractility of the torn supraspinatus muscle decreased with increasing fat infiltration. ${ }^{17,27}$ These evidences suggest postoperative contractile recovery to be associated with fat infiltration.

Our results based on the activity value showed that the sensitivity of the AR was lowest and false-positive was highest, especially in advanced fat infiltration cases. This suggests that the AR is affected by the collagenous tissue hardness and overestimates in hardness. The sensitivity of the AM-p was modest in overall but highest in advanced fat infiltration, and false-positive was higher, especially in Goutallier's stages 0 and 1 . This suggests that the outcome may become unstable if the collagenous tissue was not measured. Therefore, the sensitivity of the whole area, where the collagenous tissue was appropriately included, was highest and false-positive was lowest.

Recently, ultrasound elastography has been used to evaluate muscle quality. Alfuraih et al measured passive stiffness in quadriceps, hamstrings, and biceps brachii muscles using elastography with the $10 \mathrm{~mm}$ of ROI. ${ }^{7}$ They found significantly decreased passive stiffness in elderly groups compared to young and middle age groups. Şendur et al evaluated both passive and active stiffness in gastrocnemius muscle with 
approximately 3 to $4 \mathrm{~mm}$ of ROIs. ${ }^{8}$ They also found a strong inverse correlation between age and both passive and active stiffness. As described here, there is no consensus with respect to the size of ROI. Furthermore, they did not evaluate the advanced fat infiltrated muscle. Our study provides an implication about ROI size, namely the ROI should be set as large as possible so that we can evaluate the tissue pathological change, such as fibrosis.

Limitations in this study include a small number of patients in the subgroup analysis. Patients with stage 1 comprised the majority of the patients in this study, and consequently, those with stage $\geq 2$ were less involved because our indication for rotator cuff repair is within the earlier modified Goutallier stage, and surgical intervention is performed as early as possible. The investigation of the patients with stage $\geq 2$ needs to be considered in future studies. The torn rotator cuff muscle is not a true sarcopenia muscle. However, they showed common histopathology to sarcopenia muscle. We believe that the findings from this study could provide implications for sarcopenia. Further studies will be needed to establish ultrasound elastography measurement as a biomarker for sarcopenia.

\section{Conclusion}

The whole cross-sectional area is suitable to measure muscle activity in muscle with fat infiltration. The results in this study will provide some beneficial information when ultrasound elastography is used for the assessment of sarcopenia muscle with fat infiltration.

\section{Abbreviations}

RTE, real-time tissue elastography; AM-p, posterior portion of the anterior-middle subregion; AR, anterior region; ROI, region of interest; SR, strain ratio; ICC, intraclass correlation coefficient.

\section{Data Sharing Statement}

The datasets used and/or analyzed during the current study are available from the corresponding author on reasonable request.

\section{Ethics Approval and Consent to Participate}

As noted in the text, all protocols were approved by an institutional committee.

\section{Acknowledgments}

We would like to thank Tomohiro Uno, Ryuta Oishi, and Tadashi Koseki, whose supports made this project possible.

\section{Funding}

This study was supported by a Grant-in-Aid for JSPS fellows (Grant number 19J10699).

\section{Disclosure}

The authors declare that they have no competing interests.

\section{References}

1. Cruz-Jentoft AJ, Bahat G, Bauer J, et al. Sarcopenia: revised European consensus on definition and diagnosis. Age Ageing. 2019;48(1):16-31. doi:10.1093/ageing/afy169

2. Beeler S, Ek ET, Gerber C. A comparative analysis of fatty infiltration and muscle atrophy in patients with chronic rotator cuff tears and suprascapular neuropathy. J Shoulder Elbow Surg. 2013;22 (11):1537-1546. doi:10.1016/j.jse.2013.01.028

3. Kiyoshige Y, Watanabe E. Fatty degeneration of gluteus minimus muscle as a predictor of falls. Arch Gerontol Geriatr. 2015;60 (1):59-61. doi:10.1016/j.archger.2014.07.013

4. Meyer DC, Pirkl C, Pfirrmann CW, Zanetti M, Gerber C. Asymmetric atrophy of the supraspinatus muscle following tendon tear. $J$ Orthop Res. 2005;23(2):254-258. doi:10.1016/j.orthres.2004.06.010

5. Nakagaki K, Ozaki J, Tomita Y, Tamai S. Fatty degeneration in the supraspinatus muscle after rotator cuff tear. J Shoulder Elbow Surg. 1996;5(3):194-200. doi:10.1016/S1058-2746(05)80005-9

6. Takano Y, Kobayashi H, Yuri T, Yoshida S, Naito A, Kiyoshige Y. Fat infiltration in the gluteus minimus muscle in older adults. Clin Interv Aging. 2018;13:1011-1017. doi:10.2147/CIA.S157402

7. Alfuraih AM, Tan AL, O'Connor P, Emery P, Wakefield RJ. The effect of ageing on shear wave elastography muscle stiffness in adults. Aging Clin Exp Res. 2019;31(12):1755-1763. doi:10.1007/ s40520-019-01139-0

8. Sendur HN, Cindil E, Cerit MN, Kilic P, Gultekin II, Oktar SO. Evaluation of effects of aging on skeletal muscle elasticity using shear wave elastography. Eur $J$ Radiol. 2020;128:109038. doi:10.1016/j.ejrad.2020.109038

9. Ates F, Hug F, Bouillard K, et al. Muscle shear elastic modulus is linearly related to muscle torque over the entire range of isometric contraction intensity. J Electromyogr Kinesiol. 2015;25(4):703-708. doi:10.1016/j.jelekin.2015.02.005

10. Alfuraih AM, O'Connor P, Tan AL, Hensor E, Emery P, Wakefield RJ. An investigation into the variability between different shear wave elastography systems in muscle. Med Ultrason. 2017;19 (4):392-400. doi:10.11152/mu-1113

11. Kot BC, Zhang ZJ, Lee AW, Leung VY, Fu SN. Elastic modulus of muscle and tendon with shear wave ultrasound elastography: variations with different technical settings. PLoS One. 2012;7(8):e44348. doi:10.1371/journal.pone. 0044348

12. Hodges PW, Danneels L. Changes in structure and function of the back muscles in low back pain: different time points, observations, and mechanisms. J Orthop Sports Phys Ther. 2019;49(6):464-476. doi:10.2519/jospt.2019.8827

13. Rosatelli AL, Ravichandiran K, Agur AM. Three-dimensional study of the musculotendinous architecture of lumbar multifidus and its functional implications. Clin Anat. 2008;21(6):539-546. doi:10.1002/ ca. 20659 
14. Peterson MD, Sen A, Gordon PM. Influence of resistance exercise on lean body mass in aging adults: a meta-analysis. Med Sci Sports Exerc. 2011;43(2):249-258. doi:10.1249/MSS.0b013e3181eb6265

15. Fuchs B, Weishaupt D, Zanetti M, Hodler J, Gerber C. Fatty degeneration of the muscles of the rotator cuff: assessment by computed tomography versus magnetic resonance imaging. J Shoulder Elbow Surg. 1999;8(6):599-605. doi:10.1016/S1058-2746(99)90097-6

16. Goutallier D, Postel JM, Bernageau J, Lavau L, Voisin MC. Fatty muscle degeneration in cuff ruptures. Pre- and postoperative evaluation by CT scan. Clin Orthop Relat Res. 1994;304:78-83.

17. Yuri T, Mura N, Yuki I, Fujii H, Kiyoshige Y. Contractile property measurement of the torn supraspinatus muscle using real-time tissue elastography. J Shoulder Elbow Surg. 2018;27(9):1700-1704. doi:10.1016/j.jse.2018.02.065

18. Fujihara Y, Matsumura T, Murayama N. Development of acoustic coupler for elastography. $M E D E X$. 2011;55:40-44.

19. Kim SY, Boynton EL, Ravichandiran K, Fung LY, Bleakney R, Agur AM. Three-dimensional study of the musculotendinous architecture of supraspinatus and its functional correlations. Clin Anat. 2007;20(6):648-655. doi:10.1002/ca.20469

20. Roh MS, Wang VM, April EW, Pollock RG, Bigliani LU, Flatow EL. Anterior and posterior musculotendinous anatomy of the supraspinatus. J Shoulder Elbow Surg. 2000;9(5):436-440. doi: $10.1067 /$ mse. 2000.108387

21. Yuri T, Kuwahara Y, Fujii H, Kiyoshige Y. Functions of the subregions of the supraspinatus muscle. Clin Anat. 2017;30(3):347-351. doi:10.1002/ca.22843
22. Coleman SH, Fealy S, Ehteshami JR, et al. Chronic rotator cuff injury and repair model in sheep. J Bone Joint Surg Am. 2003;85 (12):2391-2402. doi:10.2106/00004623-200312000-00018

23. Giambini H, Hatta T, Gorny KR, et al. Intramuscular fat infiltration evaluated by magnetic resonance imaging predicts the extensibility of the supraspinatus muscle. Muscle Nerve. 2018;57(1):129-135. doi: $10.1002 /$ mus. 25673

24. Giambini H, Hatta T, Rezaei A, An KN. Extensibility of the supraspinatus muscle can be predicted by combining shear wave elastography and magnetic resonance imaging-measured quantitative metrics of stiffness and volumetric fat infiltration: a cadaveric study. Clin Biomech. 2018;57:144-149. doi:10.1016/j.clinbiomech.2018.07.001

25. Gilbert F, Klein D, Weng AM, et al. Supraspinatus muscle elasticity measured with real time shear wave ultrasound elastography correlates with MRI spectroscopic measured amount of fatty degeneration. BMC Musculoskelet Disord. 2017;18(1):549. doi:10.1186/s12891017-1911-8

26. Valencia AP, Lai JK, Iyer SR, et al. Fatty infiltration is a prognostic marker of muscle function after rotator cuff tear. Am J Sports Med. 2018;46(9):2161-2169. doi:10.1177/0363546518769267

27. Gerber C, Schneeberger AG, Hoppeler H, Meyer DC. Correlation of atrophy and fatty infiltration on strength and integrity of rotator cuff repairs: a study in thirteen patients. J Shoulder Elbow Surg. 2007;16 (6):691-696. doi:10.1016/j.jse.2007.02.122
Clinical Interventions in Aging

\section{Publish your work in this journal}

Clinical Interventions in Aging is an international, peer-reviewed journal focusing on evidence-based reports on the value or lack thereof of treatments intended to prevent or delay the onset of maladaptive correlates of aging in human beings. This journal is indexed on PubMed Central, MedLine, CAS, Scopus and the Elsevier
Bibliographic databases. The manuscript management system is completely online and includes a very quick and fair peer-review system, which is all easy to use. Visit http://www.dovepress.com/ testimonials.php to read real quotes from published authors. 